\title{
Modifications in the Stress Field of a Long Inclined Fault Caused by the Welded-Contact Boundary Conditions across the Interface between Two Elastic Half-Spaces
}

\author{
Sunita Rani ${ }^{1}$, Sarva Jit Singh ${ }^{2}$ \\ ${ }^{1}$ Department of Mathematics, Guru Jambheshwar University of Science and Technology, Hisar, India \\ ${ }^{2}$ Department of Mathematics, University of Delhi, New Delhi, India \\ Email:s_b_rani@rediffmail.com,s_j_singh@yahoo.com \\ Received October 6, 2009; revised November 18, 2009; accepted November 22, 2009
}

\begin{abstract}
In welded-contact boundary conditions, some stress components are not required to be continuous across the boundary between two elastic half-spaces. The purpose of this note is to study the modifications in the stress field of a long inclined strike-slip, dip-slip or tensile fault caused by the welded-contact boundary conditions across the interface between two elastic half-spaces. The Poisson's ratios of the two half-spaces do not appear in the stress field of a strike-slip fault. In the case of a dip-slip fault, the Poisson's ratio of the half-space in which the fault lies, has a significant influence on the stress field across the interface. However, for a tensile fault, the modification in the stress field is significantly affected by the Poisson's ratios of both the half-spaces.
\end{abstract}

Keywords: Dip-Slip Fault, Stress Field, Strike-Slip Fault, Tensile Fault, Two-Dimensional, Welded-Contact

\section{Introduction}

Elastic dislocation theory has been developed and applied for the mathematical and physical description of mechanics of earthquakes [1-4]. Extensive reviews of the application of the elastic dislocation theory to earthquake faulting problems have been given by Savage [5] and Rybicki [6]. Using this theory, stress field around earthquake faults can be calculated in a straightforward manner. Both two- and three-dimensional fault models have been used in the literature. Considering the fact that some of the faults are sufficiently long, the two-dimensional fault model is found to be adequate in many situations. Since the Earth is not homogeneous, it is necessary to consider the effect of internal boundaries on the stress field generated by earthquake faults. The knowledge of the modification of the stress field caused by internal boundaries is useful to study secondary faulting.

Bonafede and Rivalta [7] obtained a plane strain analytic solution for the displacement and stress fields produced by a long vertical tensile dislocation in the proximity of the interface between two elastic half-spaces in welded contact. After detailed numerical computations, they observed that the welded-contact boundary conditions between a hard half-space and a soft half-space are responsible for major changes in the stress field. The stress component, which is not involved in the boundary conditions, is significantly higher along the hard side of the interface. In a subsequent paper, Bonafede and Rivalta [8] derived the corresponding solution for a long vertical tensile crack. They noted that the discontinuities in the elastic parameters across the boundary act as stress concentrators for the stress component not involved in the boundary conditions.

Rivalta et al. [9] provided a plane strain analytic solution for the displacement and stress fields induced by an edge dislocation in an elastic half-space in welded contact with another elastic half-space. They found unexpected differences in the normal component of stress parallel to the interface which is not required to be continuous at the interface by the boundary conditions. This stress component shows wide regions of high stress in the harder side of the interface. Stress concentration along the interface is particularly high when the rigidity contrast is high.

In a recent paper, Rybicki and Yamashita [10] derived formulas for two-dimensional anti-plane and in-plane problems relating stresses across a plane boundary between two elastic half-spaces in welded contact, assuming a homogeneous shear stress in one of the two halfspaces. They concluded that the mechanical conditions 
related to faulting within the Earth's crust are expected to be favourable in the high rigidity media.

The purpose of this note is to study the modifications in the stress field of a two-dimensional strike-slip, dipslip or tensile fault caused by the welded-contact boundary conditions across the interface between two elastic half-spaces.

\section{Long Inclined Strike-Slip Fault}

For a two-dimensional anti-plane strain problem with reference to the $x_{2} x_{3}$-plane, the non-zero displacement and stress components are of the form

$$
\begin{aligned}
& u_{1}=u_{1}\left(x_{2}, x_{3}\right) \\
& \tau_{12}=\mu \frac{\partial u_{1}}{\partial x_{2}} \quad \tau_{13}=\mu \frac{\partial u_{1}}{\partial x_{3}}
\end{aligned}
$$

where $\mu$ denotes the rigidity.

Consider two homogeneous, isotropic, elastic halfspaces welded along the plane $x_{3}=0$. Let $x_{3}$-axis be drawn vertically downwards, $\mu_{1}$ denote the rigidity of the upper half-space $\left(x_{3}<0\right)$ and $\mu_{2}$ denote the rigidity of the lower half-space $\left(x_{3}>0\right)$. Rani and Singh [11] obtained the displacement and stress fields due to a long inclined strike-slip fault located in the lower half-space (Figure 1). The stress component $\tau_{13}$ is continuous across the interface $\left(x_{3}=0\right)$ due to the welded-contact boundary conditions. At the interface $x_{3}=0$, the stress component $\tau_{12}$ is not continuous. At any point of the interface $x_{3}=$ 0 , we have

$$
\tau_{12}^{(1)}=\frac{\mu_{1} b \sin \delta}{\pi(1+m)}\left[\frac{s_{2}}{R_{2}^{2}}-\frac{s_{1}}{R_{1}^{2}}\right]
$$

for the upper half-space, and

$$
\tau_{12}^{(2)}=\frac{\mu_{2} b \sin \delta}{\pi(1+m)}\left[\frac{s_{2}}{R_{2}^{2}}-\frac{s_{1}}{R_{1}^{2}}\right]
$$

for the lower half-space, where

$$
\begin{aligned}
m & =\frac{\mu_{1}}{\mu_{2}} \\
R_{1}^{2} & =\left(x_{2}-s_{1} \cos \delta\right)^{2}+s_{1}^{2} \sin ^{2} \delta \\
R_{2}^{2} & =\left(x_{2}-s_{2} \cos \delta\right)^{2}+s_{2}^{2} \sin ^{2} \delta
\end{aligned}
$$

$\delta$ is the dip angle, $b$ is the fault-slip and $s_{1}, s_{2}$ are shown in Figure 1. Therefore, at any point of the interface,

$$
K_{1}=\frac{\tau_{12}^{(1)}}{\tau_{12}^{(2)}}=\frac{\mu_{1}}{\mu_{2}}
$$

Equation (5) is similar to the result obtained by Rybicki and Yamashita [10] for a homogeneous anti-plane shear stress conditions in the lower half-space in welded contact with the upper half-space. We thus note that the stress ratio $K_{1}$ for a long inclined strike-slip fault is equal to the rigidity ratio. It is independent of the Poisson's ratios of the two half-spaces as well as of the dip angle $\delta$ of the fault.

\section{Long Inclined Dip-Slip Fault}

For a two-dimensional plane strain problem, the nonzero displacement and stress components are of the form

$$
\begin{aligned}
& u_{2}=u_{2}\left(x_{2}, x_{3}\right), \quad u_{3}=u_{3}\left(x_{2}, x_{3}\right) \\
& \tau_{11}=\lambda \theta \\
& \tau_{22}=\lambda \theta+2 \mu \frac{\partial u_{2}}{\partial x_{2}} \\
& \tau_{33}=\lambda \theta+2 \mu \frac{\partial u_{3}}{\partial x_{3}} \\
& \tau_{23}=\mu\left(\frac{\partial u_{2}}{\partial x_{3}}+\frac{\partial u_{3}}{\partial x_{2}}\right)
\end{aligned}
$$

where $\lambda, \mu$ are the Lamè constants and

$$
\theta=\frac{\partial u_{2}}{\partial x_{2}}+\frac{\partial u_{3}}{\partial x_{3}}
$$

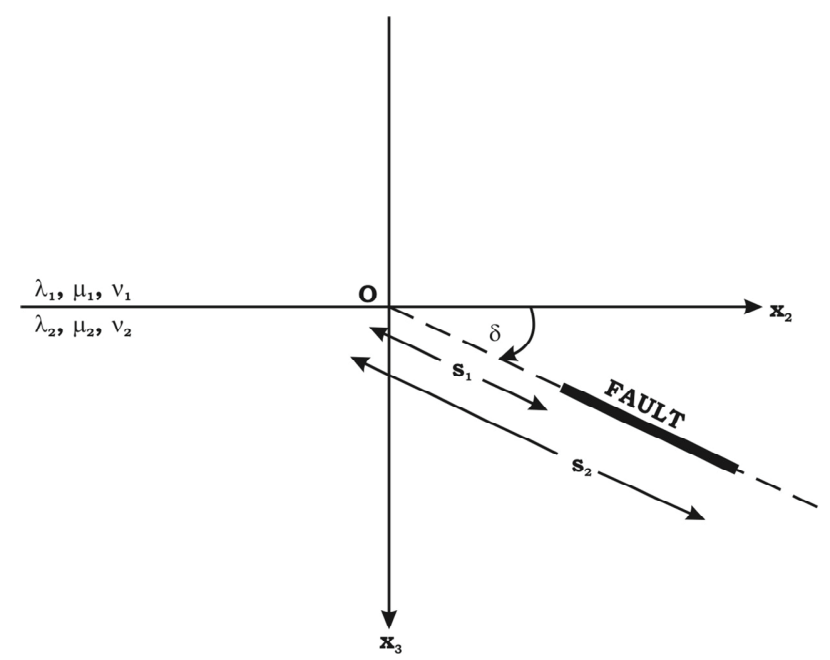

Figure 1. Geometry of a long fault lying in an elastic half-space in welded contact with another elastic half-space. The $x_{1}$-axis is taken parallel to the length of the fault and $x_{3}$-axis normal to the interface between the two half-spaces. $\delta$ is the dip angle and $s_{1}, s_{2}$ are the distances of the two edges of the fault from the origin. 
Rani and Singh [12] derived closed-form analytic expressions for the displacements and stresses caused by a long inclined dip-slip fault located in an elastic halfspace in welded contact with another elastic half-space. The stress components $\tau_{23}$ and $\tau_{33}$ are continuous across the interface as required by the welded-contact boundary conditions. In Figure 1, the origin is taken at the fault trace, that is at the point where the fault, if extended, meets the interface $x_{3}=0$. At the origin, the stress component $\tau_{22}$ is given by

$$
\tau_{22}^{(1)}=\frac{a_{2} \mu_{2} b}{\pi}\left(1+C_{2}\right) \sin 2 \delta\left(\frac{1}{s_{2}}-\frac{1}{s_{1}}\right)
$$

for the upper half-space, and

$$
\tau_{22}^{(2)}=\frac{a_{2} \mu_{2} b}{\pi}\left(1-3 C_{2}\right) \sin 2 \delta\left(\frac{1}{s_{2}}-\frac{1}{s_{1}}\right)
$$

for the lower half-space, where

$$
\begin{aligned}
a_{2} & =\frac{1}{2\left(1-v_{2}\right)} \\
C_{2} & =\frac{m-1}{1-m+2 m / a_{2}}
\end{aligned}
$$

$v_{2}$ being the Poisson's ratio of the lower half-space. Therefore, at the origin,

$$
K_{2}=\frac{\tau_{22}^{(1)}}{\tau_{22}^{(2)}}=\frac{1+C_{2}}{1-3 C_{2}}=\frac{m\left(1-v_{2}\right)}{1-m v_{2}}=1-\frac{1-m}{1-m v_{2}}
$$

We observe that the stress ratio $K_{2}$ for a long inclined dip-slip fault depends upon the rigidity contrast $m=$ $\mu_{1} / \mu_{2}$ and the Poisson's ratio $v_{2}$ of the lower half-space. It is independent of the Poisson's ratio $v_{1}$ of the upper half-space as well as of the dip angle $\delta$. From (11), if $m<1$, then $K_{2}<1$. This implies that the stress near the interface generated in the upper half-space is less than the stress near the interface in the lower half-space in which the fault lies. On the other hand, if $1<m<1 / v_{2}$, then $K_{2}>1$, implying that the stress generated near the interface in the upper half-space is more than the stress near the interface in the lower half-space. For $m=v_{2}^{-1}$, the stress $\tau_{22}^{(2)}$ at the origin vanishes.

It is interesting to compare (11) with the corresponding result of Rybicki and Yamashita [10] which, in our notation, is reproduced below:

$$
K_{2 R}=\frac{\tau_{22}^{(1)}}{\tau_{22}^{(2)}}=m+v_{1}(m-1)
$$

where $v_{1}$ is the Poisson's ratio of the upper half-space. The stress ratio $K_{2 \mathrm{R}}$ derived by Rybicki and Yamashita
[10] depends upon the rigidity contrast $m$ and the Poisson's ratio of the upper half-space. It is independent of the Poisson's ratio of the lower half-space. It may be noted that while Rybicki and Yamashita [10] considered a homogeneous shear stress acting on an inclined plane in the lower medium, we have considered the stress field of a long inclined dip-slip fault in the lower half-space.

Figure 2 shows the variation of $K_{2}$ with $m$ for $v_{2}=0.1$, $0.2,0.3,0.4$. Since $K_{2}$ is independent of $v_{1}$, for $m=1$, we have $K_{2}=1$ corresponding to the case when the two half-spaces have identical elastic parameters. In the range $0<m<1$ (Figure 2(a)), we note that $K_{2}<1$ and, for a given $m, K_{2}$ decreases as $v_{2}$ increases. The compressibility is given by $\frac{3(1-2 v)}{2(1+v) \mu}$. Therefore, in the range $0<m<1$, for a given $m, K_{2}$ increases as the compressibility of the lower half-space increases. In contrast, in the range $1<m<2$ (Figure 2(b)), $K_{2}>1$ and, for a

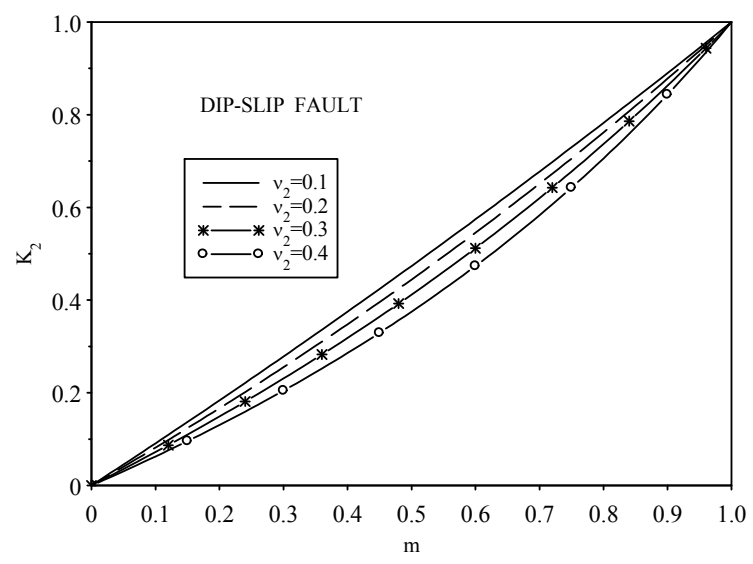

(a)

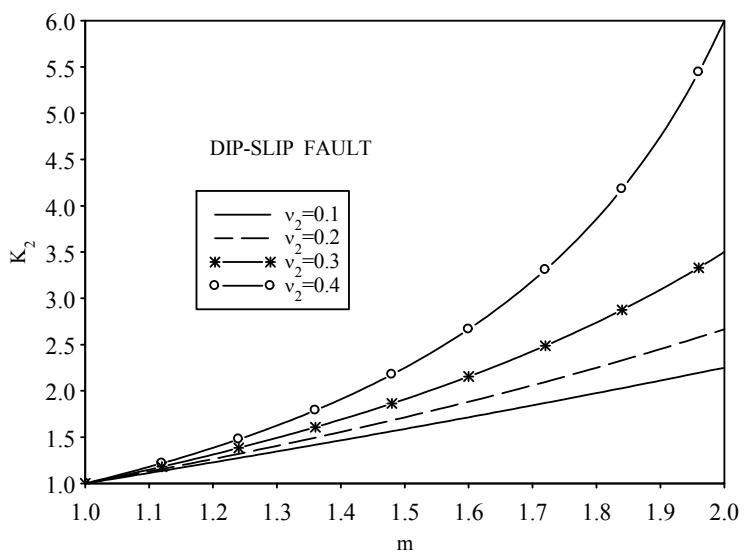

(b)

Figure 2. Variation of the stress ratio $K_{2}$ for a long dip-slip fault with the rigidity ratio $m=\mu_{1} / \mu_{2}$ for four values of the Poisson's ratio $v_{2}$ of the lower half-space for (a) $\mathbf{0} \leq \boldsymbol{m} \leq \mathbf{1}$; (b) $\mathbf{1} \leq \boldsymbol{m} \leq \mathbf{2}$. 
given $m, K_{2}$ decreases as the compressibility of the lower half-space increases. Thus the most favourable elastic conditions for the generation of higher stresses in the upper half-space (near the interface) occur when the fault lies in the softer half-space with small compressibility.

\section{Long Inclined Tensile Fault}

This is also a plane strain problem. Kumar et al. [13] obtained closed-form, analytic expressions for the displacements and stresses for a long inclined tensile fault situated in an elastic half-space in welded contact with another elastic half-space. At the origin, the stress component $\tau_{22}$ is given by

$$
\tau_{22}^{(1)}=\frac{a_{2} \mu_{2} b}{2 \pi}\left(1+3 C_{1}+C_{2}\right)\left(\frac{1}{s_{2}}-\frac{1}{s_{1}}\right)
$$

for the upper half-space, and

$$
\tau_{22}^{(2)}=-\frac{a_{2} \mu_{2} b}{2 \pi}\left(3+C_{1}+3 C_{2}\right)\left(\frac{1}{s_{2}}-\frac{1}{s_{1}}\right)
$$

for the lower half-space, where

$$
C_{1}=\frac{2 m}{a_{2}\left(1-m-2 / a_{1}\right)}, a_{1}=\frac{1}{2\left(1-v_{1}\right)}
$$

$v_{1}$ being the Poisson's ratio in the upper half-space. Therefore, at the origin,

$$
K_{3}=\frac{\tau_{22}^{(1)}}{\tau_{22}^{(2)}}=-\frac{1+3 C_{1}+C_{2}}{3+C_{1}+3 C_{2}}=\frac{2 m+v_{1}-3 m v_{2}}{2-3 v_{1}+m v_{2}}
$$

It may be noted that the stress ratio $K_{3}$ for a long inclined tensile fault depends upon the rigidity ratio $m$ and the Poisson's ratios $v_{1}, v_{2}$. It is independent of the dip angle $\delta$.

Figure 3 shows the variation of $K_{3}$ with $m$ for $v_{1}=v_{2}=$ $0.1,0.2,0.3,0.4$. We note that, $K_{3}<1$ for $0<m<1$ (Figure 3(a)) and $K_{3}>1$ for $1<m<4$ (Figure 3(b)). When $m=1$, the two half-spaces have identical properties and $K_{3}=1$. For a given $v_{1}$, the variation of $K_{3}$ with $m$ for $v_{2}=0.1,0.2,0.3,0.4$ is shown in Figure 4. In this case, $m=1$ does not imply that the two half-spaces are identical because of the difference in the values of $v_{1}$ and $v_{2}$. Here, we note that for a given $m, K_{3}$ increases as the compressibility of the lower half-space increases. For a given $v_{2}$, the variation of $K_{3}$ with $m$ for $v_{1}=0.1,0.2,0.3$, 0.4 is shown in Figure 5. For a given $m, K_{3}$ increases as the compressibility of the upper half-space decreases. Figure 6 shows the variation of $K_{3}$ with $v_{2}$ for a given value of $v_{1}$. In contrast, Figure 7 shows the variation of $K_{3}$ with $v_{1}$ for a given value of $v_{2}$. Since, for an elastic material of given rigidity, the compressibility decreases as the Poisson's ratio increases, Figures 6 and 7 taken together indicate that the most favourable elastic condi-

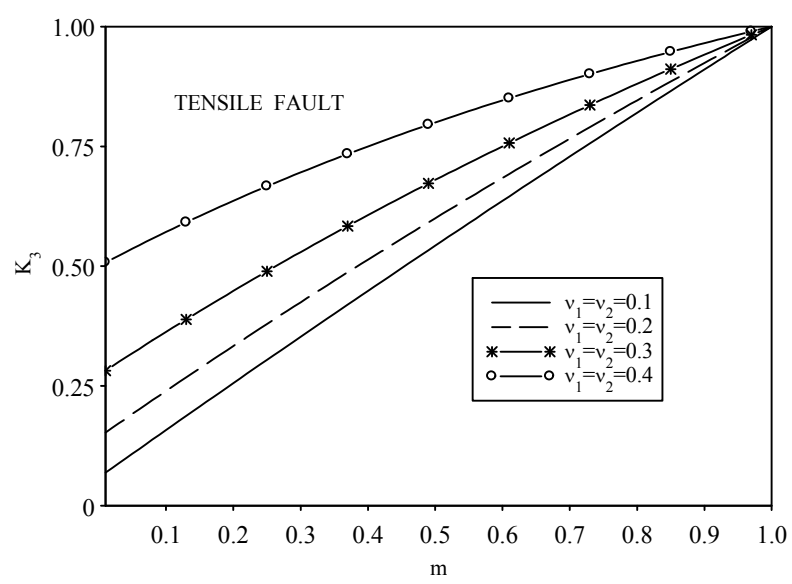

(a)

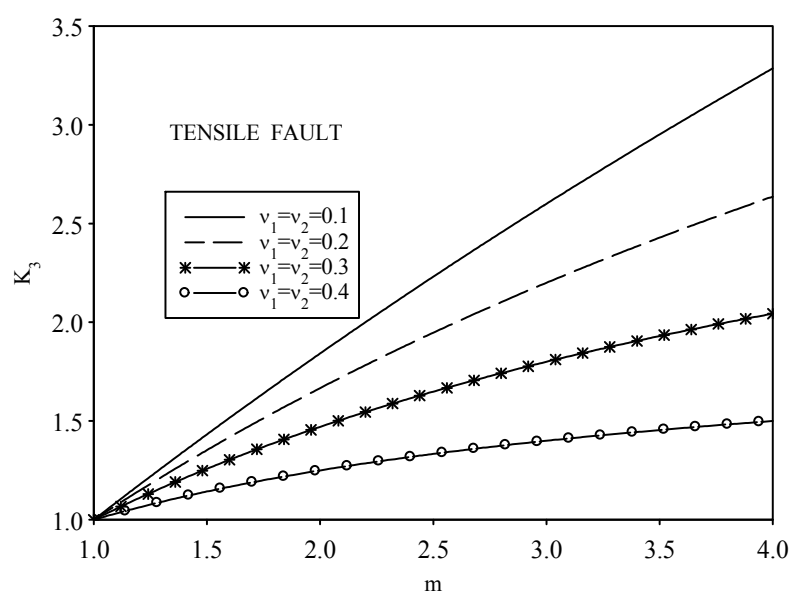

(b)

Figure 3. Variation of the stress ratio $K_{3}$ for a long tensile fault with the rigidity ratio $m$ for (a) $0 \leq m \leq 1$; (b) $\mathbf{1} \leq \boldsymbol{m} \leq \mathbf{4}$.

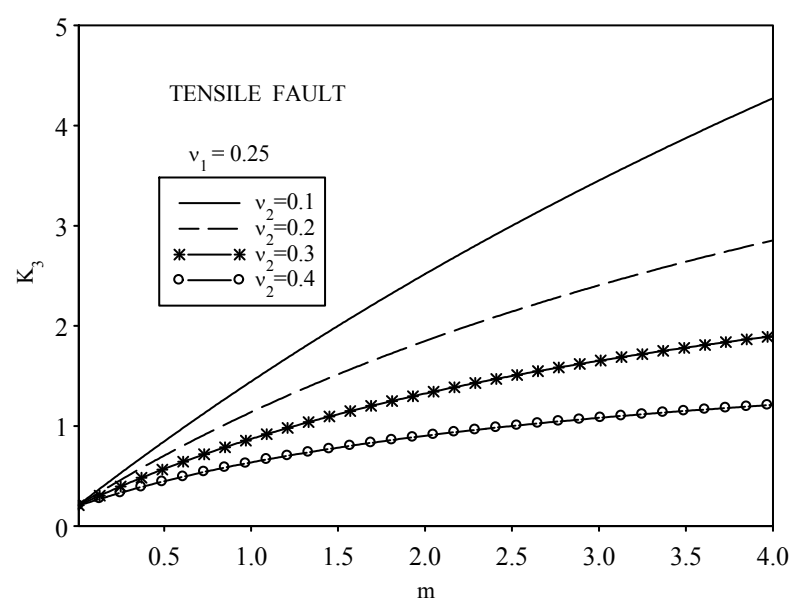

Figure 4. Showing the effect of the Poisson's ratio $v_{2}$ of the lower half-space on the variation of the stress ratio $K_{3}$ for a tensile fault with the rigidity ratio $m$. 


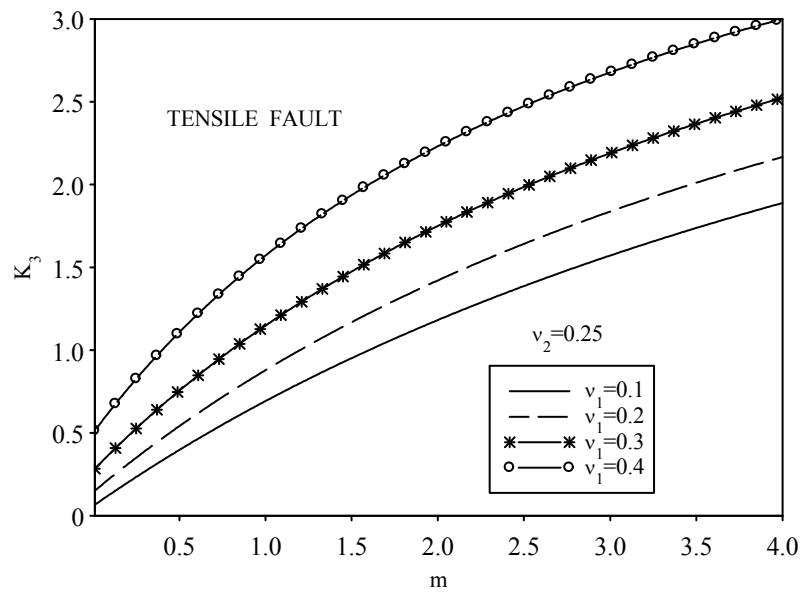

Figure 5. Showing the effect of the Poisson's ratio $v_{1}$ of the upper half-space on the variation of the stress ratio $K_{3}$ for a tensile fault with the rigidity ratio $m$.

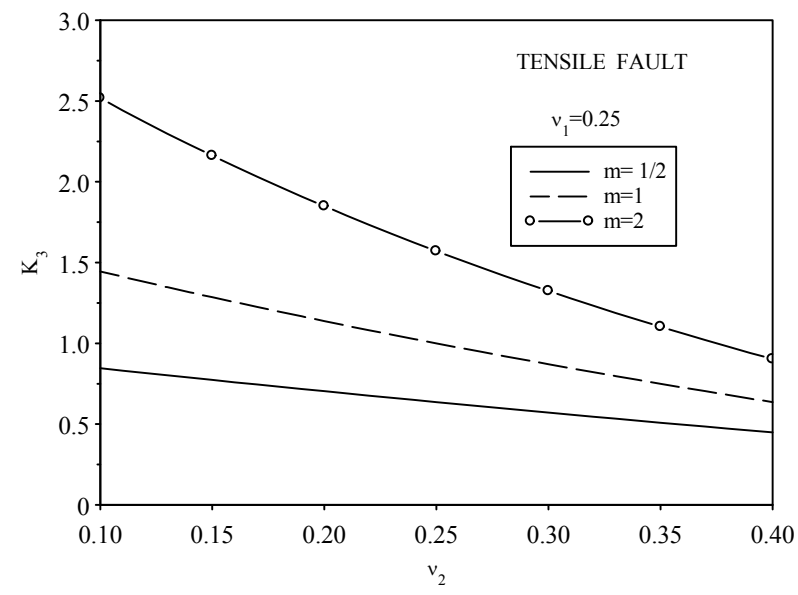

Figure 6. Variation of the stress ratio $K_{3}$ for a tensile fault with the Poisson's ratio $v_{2}$ of the lower half-space.

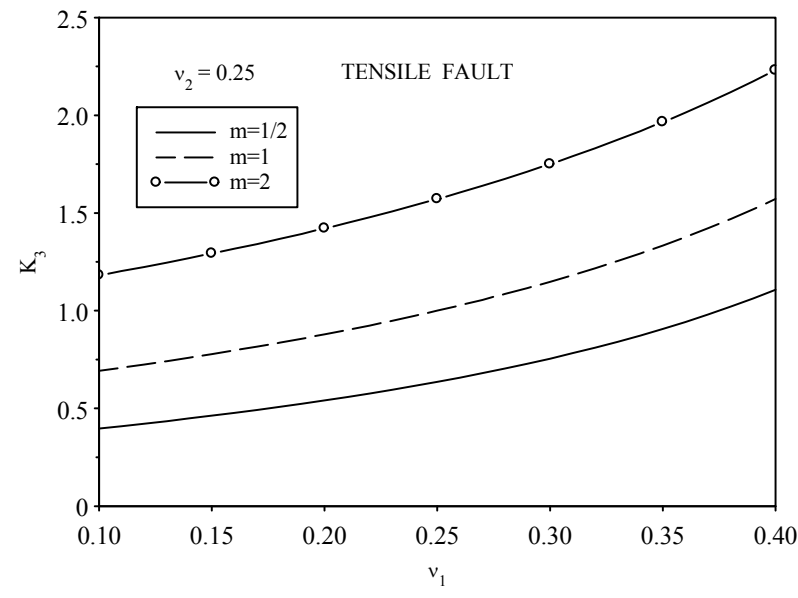

Figure 7. Variation of the stress ratio $K_{3}$ for a tensile fault with the Poisson's ratio $v_{1}$ of the upper half-space. tions for generation of high stresses in the upper halfspace (near the interface) occur when the tensile fault lies in the softer half-space, the compressibility of the upper half is least and the compressibility of the lower halfspace (in which the fault lies) is greatest.

\section{Conclusions}

In general, when studying the static field in two elastic half-spaces in contact with source in one of the halfspaces, it is assumed that the half-spaces are in welded contact. In welded-contact boundary conditions, the displacement vector and the stress vector across the boundary are assumed to be continuous at the boundary. Consequently, some of the stress components are not required to be continuous at the boundary. We have studied the modifications in the stress field of a two-dimensional inclined strike-slip, dip-slip or tensile fault caused by the welded-contact boundary conditions across the boundary between the two elastic half-spaces. The results of the study can be summarised as under. It is assumed that the boundary between the half-spaces is taken as the $x_{3}=0$ plane and the fault is striking in the $x_{1}$-direction.

1) In the case of a long inclined strike-slip fault, the shear stress $\tau_{13}$ is required to be continuous across the interface by the boundary conditions. However, there is no restriction on the shear stress $\tau_{12}$. We find that the ratio of the values of the shear stress $\tau_{12}$ at the interface when approached from the two half-spaces is equal to the rigidity ratio. It is independent of the dip angle of the fault or the Poisson's ratios of the two half-spaces.

2) In the case of a long inclined dip-slip fault, the normal stress $\tau_{33}$ and the shear stress $\tau_{13}$ are required to be continuous. There is no restriction on the normal stresses $\tau_{11}$ and $\tau_{22}$. We find that the ratio of the normal stress $\tau_{22}$ at the interface when approached from the two half-spaces depends on the rigidity ratio and the Poisson's ratio of the half-space in which the fault lies. It is independent of the Poisson's ratio of the other half-space as well as of the dip angle of the fault. The most favourable elastic conditions for generation of large stresses near the interface in the other half-space occur when the dip-slip fault lies in the softer half-space with small compressibility.

3 ) In the case of a long inclined tensile fault also, $\tau_{33}$ and $\tau_{13}$ are continuous. We find that the ratio of the normal stress $\tau_{22}$ at the interface depends upon the rigidity ratio as well as upon the Poisson's ratios of the two half-spaces. It is independent of the dip angle. The most favourable elastic conditions for generation of large stresses near the interface in the half-space, in which the fault does not lie, occur when the tensile fault lies in the 
softer half-space, the compressibility of the half-space with fault is large and the compressibility of the other half-space is small.

\section{Acknowledgment}

The authors thank the referee for the improvement of the paper. SJS thanks the Indian National Science Academy for financial support under its Senior Scientist Scheme.

\section{References}

[1] J. A. Steketee, "On Volterra's disclocations in a semiinfinite elastic medium," Canadian Journal of Physics, Vol. 36, pp. 192-205, 1958.

[2] J. A. Steketee, "Some geophysical applications of the elasticity theory of dislocation," Canadian Journal of Physics, Vol. 36, pp. 1168-1198, 1958.

[3] T. Maruyama, "Statical elastic dislocations in an infinite and semi-infinite medium," Bulletin of Earthquake Research Institute, Vol. 42, pp. 289-368, 1964.

[4] T. Maruyama, "On two-dimensional elastic dislocations in an infinite and semi-infinite medium," Bulletin of Earthquake Research Institute, Vol. 44, pp. 811-871, 1966.

[5] J. C. Savage, "Dislocations in seismology," In: F. R. N. Nabarro, Ed., Dislocations in Solids, Moving Dislocations, Amsterdam, Vol. 3, pp. 251-339, 1980.
[6] K. Rybicki, "Dislocations and their geophysical applications," In: R. Tiesseyre, Ed., Continuum Theories in Solid Earth Physics, Elsevier, Amsterdam, pp. 18-186, 1986.

[7] M. Bonafede and E. Rivalta, "The tensile dislocation problem in a layered elastic medium," Geophysical Journal International, Vol. 136, pp. 341-356, 1999.

[8] M. Bonafede and E. Rivalta, "On tensile cracks close to and across the interface between two welded elastic half-spaces," Geophysical Journal International, Vol. 138, pp. 410-434, 1999.

[9] E. Rivalta, W. Mangiavillano and M. Bonafede, "The edge dislocation problem in a layered elastic medium," Geophysical Journal International, Vol. 149, pp. 508-523, 2002.

[10] K. R. Rybicki and T. Yamashita, "Constrains on stresses in isotropic homogeneous infinite half-spaces being in welded contact: 2D anti-plane and in-plane cases," Acta Geophysica, Vol. 56, pp. 286-292, 2008.

[11] S. Rani and S. J. Singh, "Residual elastic field in two welded half-spaces caused by a long inclined strike-slip fault," Proceedings of the Indian National Science Academy, Vol. 59, pp. 455-464, 1993.

[12] S. Rani and S. J. Singh, "Static deformation of two welded half-spaces due to dip-slip faulting," Proceedings of the Indian Academy of Sciences (Earth and Planetary Science), Vol. 101, pp. 269-282, 1992.

[13] A. Kumar, S. J. Singh and J. Singh, "Deformation of two welded elastic half-spaces due to a long inclined tensile fault," Journal of Earth System Science, Vol. 114, pp. 97-103, 2005. 